\title{
EL CONOCIMIENTO NORMATIVO DE FOMENTO AL TURISMO DE PARTE DE SUS OPERADORES Y SU RELACIÓN CON EL IMPULSO AL DESARROLLO SOSTENIBLE DE PARTE DE LAS AUTORIDADES LOCALES Y REGIONALES DE TACNA LEGAL KNOWLEDGE TO TOURISM DEVELOPMENT OF PART OF THEIR OPERATORS AND THEIR RELATION TO THE URGE TO SUSTAINABLE DEVELOPMENT OF LOCAL AUTHORITIES AND REGIONALS TACNA
}

Julio Fidel Muñoz Flores ${ }^{1}$

\begin{abstract}
RESUMEN
El turismo mejora el intercambio cultural y es una necesidad para visitantes y visitados, debido a la satisfacción que otorga a los visitantes y la generación de ingresos en la región visitada; siendo importante que se conozcan y apliquen el conjunto de normas que promueven el turismo.

Sin embargo; se presenta un aislamiento de los hechos con la normatividad de promoción del turismo.

Objetivo: El objetivo es relacionar la incidencia del conocimiento normativo de fomento al turismo de parte de sus operadores con el impulso al desarrollo sostenible de parte de las autoridades competentes de Tacna.

Método: La presente investigación es de tipo descriptivo correlacional, su diseño es no experimental; la población de estudio fue (17) operadores turísticos de Tacna.

Resultados: Se ha encontrado que existe una relación directa y significativa.

Conclusiones: Que entre el conocimiento normativo de fomento al turismo de los operadores y el impulso al desarrollo sostenible de las autoridades de Tacna tienen una relación directa y significativa.
\end{abstract}

PALABRAS CLAVE: normatividad, promoción, inversión, actores, capacitación y turismo.

\begin{abstract}
Tourism better cultural exchange and is a must for visitors and visited, due to satisfaction given to visitors and the generation of income in the region visited; still important to know and apply the set of standards that promote tourism in Tacna.

However; isolation of the facts is presented with regulations for the promotion of tourism.

Objetivo: The objective is to relate the incidence of tourism development normative knowledge by operators to the promotion of sustainability by the competent authorities of Tacna.

Method: This research is descriptive-correlational study, its design is not experimental; the study population was (17) tour operators of Tacna.

Result: Found that there is a direct and meaningful relationship.

Conclusion: That between regulatory knowledge to promote tourism of the operators and the impulse sustainable development Tacna authorities have a direct and significant.
\end{abstract}

KEYWORDS: regulations, promotion, investment, actors, training and tourism.

\footnotetext{
Tesis de postgrado para optar el Grado de Mg. en Derecho con Mención en Derecho Civil y Comercial. Escuela de Postgrado - Universidad Privada de Tacna. E-mail:juliofmf2@hotmail.com
} 


\section{INTRODUCCIÓN}

La Constitución Política del Perú establece un estado social de derecho, que mediante la libertad de la actividad económica, se desarrollen los sectores productivos del país y lograr una mejor redistribución de los factores productivos.

La Región Tacna es una ciudad geográficamente ubicada en la parte sur del Perú, donde su principal actividad es la comercial, pero que a través de los años tiene incrementos y decrementos en dichas actividad; por lo que es necesario fomentar otras actividades como el turismo en todas sus formas, que podría diversificar las actividades ocupacionales de Tacna.

El movimiento comercial de los residentes; así como de la población que ha instalado empresas comerciales en Tacna como fomento al turismo y desarrollo sostenible de la región, demuestran que la microempresa es la que tiene mayores márgenes de actividad laboral, no existiendo cantidad significativa de medianas y grandes empresas, por lo que la población clama por mayores inversiones en diferentes ámbitos de la actividad económica, siendo una de ellas el turismo.

El Perú cuenta con la Ley General del Turismo (29408) que rige la actividad turística. Tiene como objetivo promover, incentivar y regular el desarrollo sostenible de la actividad turística en los tres niveles de gobierno: Nacional, Regional y Local, en coordinación con los distintos actores vinculados al sector y regular la actividad turística, como factor de desarrollo económico y social del país; así como, establecer los mecanismos de participación y concertación de los sectores públicos y privados.

Frente a esta situación existe actualmente la ausencia de condiciones de capacitación, servicio e infraestructura que favorezca el fomento al turismo como desarrollo sostenible de la región.

La constitución política del Perú, permite la intervención del estado en la economía y consagra a la promoción de la productividad y de la competitividad empresarial, con relevancia la generación del empleo; entonces cabe fomentar el turismo por parte del sector público y privado con el fin de lograr productividad en una economía social de mercado hacia el desarrollo sostenible de la región Tacna; y aparte de otros factores que podrían estar presente, se plantea la Pregunta de investigación: ¿existe una relación directa y significativa entre el conocimiento normativo de fomento al turismo de parte de sus operadores y el impulso al desarrollo sostenible de parte de las autoridades regionales y locales de Tacna?.

\section{Objetivo General}

Relacionar la incidencia del conocimiento normativo de fomento al turismo de parte de sus operadores con el impulso al desarrollo sostenible de parte de las autoridades regionales y locales de Tacna.

\section{Hipótesis}

Existe una relación directa y significativa entre el conocimiento normativo de fomento al turismo de parte de sus operadores y el impulso al desarrollo sostenible de parte de las autoridades regionales y locales de Tacna.

\section{Justificación}

En los últimos 50 años, Tacna, ha vivido un devenir constante de épocas de apogeo y de declive comercial, con el vaivén de los incrementos y decrementos de índices turísticos y es que la ubicación estratégica de Tacna por ser zona de frontera ha generado un constante flujo turístico de carácter internacional especialmente con la ciudad de Arica-chile, aspecto que está vinculado con el posicionamiento geopolítico fronterizo consolidado progresivamente y que debe ser aprovechado para fomentar el turismo como desarrollo sostenible de la región.

Ante esta situación regulada mediante leyes y normas legales, cuyo propósito es el desarrollo y fomento del turismo, existe debilidades en su aplicación como problemática que amerita una investigación seria y objetiva que permita comprobar la necesidad de establecer el nivel del conocimiento normativo sobre fomento al turismo entre operadores turísticos y entidades gubernamentales involucrados en la actividad turística, explicando los hechos, la existencia de normas sobre turismo y la necesidad de promover y aplicarlas como eje significativo para contribuir al desarrollo sostenible de la región de Tacna.

\section{MATERIALES Y MÉTODO}

Se estableció como un estudio de tipo descriptivo correlacional y su diseño es no experimental, teniendo como estudio al conjunto de operadores turísticos de Tacna.

El procedimiento de levantamiento de información, se desarrolló conforme a la siguiente secuencia: Se estableció la muestra objeto de estudio (17 Operadores turísticos), se elaboró las preguntas respectivas (26 subindicadores, valorados conforme a la escala de Rensis Likert), se puso a prueba las preguntas y se redactó las preguntas definitivas, se aplicó el cuestionario, se analizó 
las respuestas y se integró los resultados al presente trabajo de investigación (utilizando el programa de aplicación estadística SPSS) presentando y analizando los resultados.

\section{SE PROCEDIÓ A TRATAR LOS DATOS ATENDIENDO LA SIGUIENTE SECUENCIA:}

- El uso de la estadística descriptiva para el procesamiento de los datos recolectados.

- Se determinó la confiabilidad del instrumento mediante el estadístico Alpha de Cronbach.

Se desarrolló la recopilación de datos a través de una encuesta dirigido a operadores de turismo de Tacna.

El procesamiento manual y computarizado, se desarrolló mediante las siguientes acciones: recepción, acopio, clasificación y tabulación de los datos.

- Se utilizó el programa de aplicación computarizado Word, Excel y Pasw-SPSS (estructurando la base de datos e introduciendo los datos obtenidos)

Se realizó el análisis de la información obtenida conforme al siguiente detalle:

- Se descompuso en cuadros estadísticos con indicadores referidos al turismo. Se comparó los indicadores de cada variable específica independiente y dependiente.

- Se procedió a probar la hipótesis mediante la prueba estadística Chi cuadrado para verificar la correlación, a través de la prueba de dependencia, para conocer si están relacionadas las variables.

- Se utilizó el programa SPSS20, para conocer la correlación entre las variables de estudio y obtener cuadros y figuras estadísticas.

- Los datos obtenidos en la investigación, se organizó y procesó en forma computarizada, con el propósito de presentar la información de forma ordenada, clara y sencilla.

\section{RESULTADOS}

Para la valoración e interpretación de los resultados se agrupo en indicadores, para la variable CONOCIMIENTO NORMATIVO DE FOMENTO AL TURISMO, se considero los siguientes indicadores: Funcionamiento de la red de protección al turista, Relación con la policía de turismo, Gestión de la información y orientación al turista, Articulación de intervención entre las entidades del poder ejecutivo regional y el ministerio público para la protección y defensa del turista; y para la variable IMPULSO AL DESARROLLO SOSTENIBLE DE LA REGIÓN TACNA, los indicadores siguientes: Factores de promoción turística, Factores estratégicos del comercio, Planeamiento estratégico, Desarrollo regional.
- La interpretación de la aplicación del estadístico Alpha de Cronbach, es que entre más cercano este a 1, refleja una baja dispersión de las respuestas recogidas; y el análisis de datos se basó en la escala de Likert que posteriormente se proceso mediante el programa estadístico SPSS.20.

Los estadísticos de fiabilidad para la Variable CONOCIMIENTO NORMATIVO DE FOMENTO AL TURISMO, se obtuvo un valor de $\mathbf{0 , 8 2 8}$ y para la Variable IMPULSO AL DESARROLLO SOSTENIBLE DE LA REGIÓN TACNA, se obtuvo un valor de 0,896 que implica que la dispersión de las respuestas es baja, por tanto, la fiabilidad del instrumento aplicado es adecuado.

Los niveles de valoración para las variables Conocimiento normativo de fomento al turismo y variable dependiente Impulso al desarrollo sostenible de la Región son:

\section{CUADRO 01 NIVELES DE VALORACIÓN}

\begin{tabular}{|l|c|}
\hline \multicolumn{1}{|c|}{ ESCALA } & INTERVALO \\
\hline MUY BAJO & $13-22$ \\
\hline BAJO & $23-32$ \\
\hline REGULAR & $33-42$ \\
\hline ALTO & $43-52$ \\
\hline MUY ALTO & $53-65$ \\
\hline
\end{tabular}

Fuente: Escala de LIKERT. Elaboración: Propia.

\section{CUADRO 02}

RESULTADOS POR INDICADOR: Conocimiento normativo de fomento al turismo

\begin{tabular}{|c|c|}
\hline INDICADORES & Media \\
\hline $\begin{array}{l}\text { Funcionamiento de la red de } \\
\text { protección al turista. }\end{array}$ & 3,0784 \\
\hline Relación con la policía de turismo. & 2,0882 \\
\hline $\begin{array}{l}\text { Gestión de la información y } \\
\text { orientación al turista }\end{array}$ & 3,2353 \\
\hline $\begin{array}{l}\text { Involucramiento de los gremios del } \\
\text { sector privado del turismo. }\end{array}$ & 2,5686 \\
\hline $\begin{array}{l}\text { Articulación de intervención entre } \\
\text { las entidades del poder ejecutivo } \\
\text { regional y el ministerio público para } \\
\text { la protección y defensa del turista. }\end{array}$ & 2,2647 \\
\hline
\end{tabular}

Fuente: Cuestionario de "Conocimiento normativo de fomento al turismo"; Reporte SPSS. Elaboración: Propia. 
Los resultados por indicador conforme a la Escala de Likert del 1 a 5 , de la variable independiente Conocimiento normativo de fomento al turismo, hacen referencia como menor valor medio $(2,09)$ al indicador denominado "Relación con la policía de turismo"; que implica que los operadores turísticas de Tacna perciben como inadecuado el nivel de relación existente con la policía de turismo; precisando dentro de ello el aspectos a mejorar: El impulsar el que el Gobierno Regional y/o Local desarrollen Proyectos de Inversión Pública que fomenten el turismo y estrechar la relación con la Policía de Turismo para proporcionar un mejor servicio a los visitantes a Tacna. Como valor medio máximo $(3,24)$, se encontró al indicador denominado "Gestión de la información y orientación al turista"; que implica que los operadores turísticas de Tacna perciben como REGULAR el nivel de gestión de la información y orientación a los turistas, siendo el aspecto a mejorar el impulsar que el Gobierno Regional y/o Local utilice las Tecnologías de la Información y de la Comunicación (TIC) para el fomento de la actividad turística regional.

CUADRO 03

RESULTADO GLOBAL: Conocimiento normativo de fomento al turismo

\begin{tabular}{|l|c|c|c|}
\hline \multicolumn{4}{|c|}{ Estadisticos descriptivos } \\
\hline VARIABLE & N & Media & $\begin{array}{c}\text { Desv. } \\
\text { tip. }\end{array}$ \\
\hline $\begin{array}{l}\text { Conocimiento } \\
\text { normativo de } \\
\text { fomento al turismo }\end{array}$ & 17 & 35,35 & 5,195 \\
\hline
\end{tabular}

Fuente: Cuestionario de "Conocimiento normativo de fomento al turismo". Reporte del SPSS 20. Elaboración: Propia.

Del análisis se encontró que el valor medio global fue de 35,35 y una desviación típica de 5,195, que al ubicarlo en la escala de valoración previamente determina, se concluye que cae en el nivel denominado "Conocimiento normativo de fomento al turismo regular". Por tanto, los operadores turísticos de Tacna consideran que su nivel de conocimiento de la normatividad afín al fomento al turismo es REGULAR.

\section{CUADRO 04 \\ RESULTADOS POR INDICADOR: Impulso al desarrollo sostenible de la región}

\begin{tabular}{|l|c|}
\hline \multicolumn{1}{|c|}{ INDICADORES } & Media \\
\hline Factores de promoción turística. & 2,6176 \\
\hline Factores estratégicos del comercio. & 3,3725 \\
\hline Planeamiento estratégico. & 2,5098 \\
\hline Desarrollo regional. & 2,6275 \\
\hline
\end{tabular}

Fuente: Cuestionario de "Impulso al desarrollo sostenible de la región". Reporte SPSS Elaboración: Propia.

Los resultados encontrados hacen referencia al indicador denominado "Planeamiento estratégico" cuyo valor medio fue de 2,51 con una desviación típica de 0,746; que implica que los operadores turísticas de Tacna perciben como inadecuado el nivel de planificación estratégico aplicado en la región de Tacna; Al analizar por pregunta se precisan los aspectos a mejorar: El ser considerado como agente participante en la formulación e implementación del plan de desarrollo regional de turismo, y el impulso del Gobierno Regional y/o Local en el desarrollo turístico de Tacna.

\section{CUADRO 05}

RESULTADO GLOBAL: Impulso al desarrollo sostenible de la región

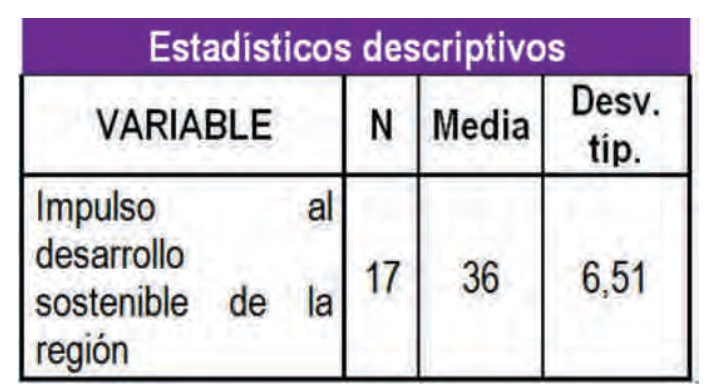

Fuente: Cuestionario de "Impulso al desarrollo sostenible de la región". Reporte del SPSS 20. Elaboración: Propia.

Ubicación en la escala de valoración (36): Regular. Por tanto, los operadores turísticos de Tacna consideran que es REGULAR el impulso de las entidades del Estado regionales y locales con respecto al turismo. 


\section{DISCUSIÓN}

Se utilizó el Coeficiente de Correlación de Pearson; siendo el reporte del SPSS 20, teniendo mediante la contrastación de la hipótesis lo siguiente:

\section{CUADRO 06 \\ CONTRASTACIÓN DE HIPÓTESIS}

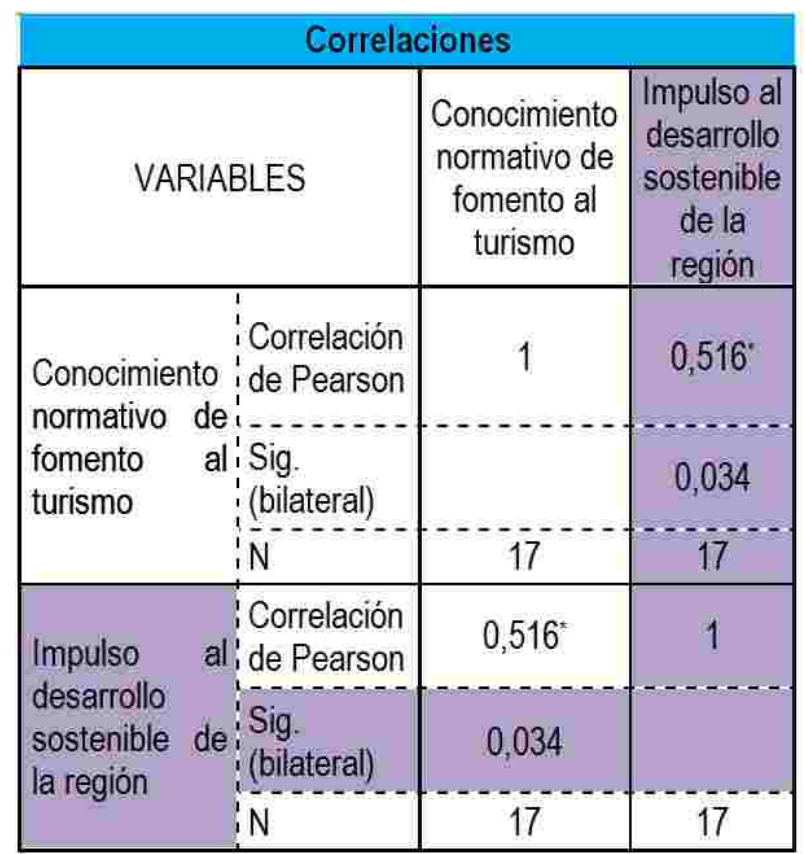

Fuente: Cuestionario de "Impulso al desarrollo sostenible de la región". Reporte SPSS. Elaboración: Propia.

La correlación es significante al nivel 0,05 (bilateral).

- Ho:No existe relación entre las variables

- H1: Existe relación entre las variables

El valor fue $\mathrm{r}=0,516$ (valor de $\mathrm{p}=0,034$ ); por lo tanto, se rechaza $\mathrm{H} 0$; es decir, que sí existe una relación directa y significativa entre el conocimiento normativo de fomento al turismo de parte de sus operadores y el impulso al desarrollo sostenible de parte de las autoridades regionales y locales de Tacna.

\section{CONCLUSIÓN}

La constitución política del Perú regula y promueve las actividades económicas con el objeto de lograr bienestar social.

Región Tacna una zona fronteriza del Perú, tiene dentro sus actividades económicas al comercio y turismo comercial sus principales actividades; por lo que para fomentar dicha actividad existe un conjunto de normatividad que deben ser ejecutadas por el Gobierno nacional, regional y local con alcance, entre otros, a los operadores turísticos.

Se encontró que existe una relación directa y significativa entre el conocimiento normativo de fomento al turismo de parte de sus operadores y el impulso al desarrollo sostenible de parte de las autoridades regionales y locales de Tacna.

Se infiere que, a mayor conocimiento normativo de los operadores turísticos y correcta aplicación de los mismos de parte de las autoridades de Tacna, mayor será su potencialidad para fomentar el desarrollo sostenible.

\section{RECOMENDACIONES}

Ejecutar el conjunto de normatividad que guarda relación con el fomento al turismo.

- Lograr mayor participación de la Dirección Regional de Turismo-Tacna ofreciendo un espacio de poder trabajar conjuntamente para realizar investigaciones de mercado, llevar registro de estadísticas, planificar eventos a fin de fomentar el turismo.

- Implementar un programa de concientización, promoción y difusión de los beneficios del turismo para lograr inversiones y colaboración de los operadores turísticos.

- Aplicar la normatividad que dispone el fomento y ejecución de inversión en infraestructura básica, orientado a localizar, diversificar y especializar el turismo.

\section{Considerar lo siguiente:}

- Incluir en el currículo académico de las instituciones educativas y centros de estudios superiores el estudio de las normas legales que impulsen el turismo a fin de que estén en capacidad de organizar, planificar y conducir empresas turísticas; asimismo, en los colegios profesionales, empresas, asociaciones universidades, con demanda nacional e internacional, estimular la actualización, capacitación y entrenamiento constante donde la capacitación es la herramienta principal para ser competitiva $\mathrm{y}$ contribuir así al desarrollo sostenible de la región.

- Impulsar la actividad turística por tipo en la cadena productiva de servicios (Agencias, restaurantes, transportes); generando empleo y comprometerse con el uso sostenible del medio ambiente. 
- Incluir dentro de la normatividad de gestión, ya sea en su Plan Estratégico ó Plan de Desarrollo Regional mayores alianzas estratégicas con el empresariado privado.

- Se sugiere que las empresas vinculadas al sector turístico en la región de Tacna participen en un proceso de integración vertical realizando dichas alianzas para captar a una mayor cantidad de clientes nacionales y extranjeros a costos más bajos y de manera simultánea iniciar una campaña de posicionamiento de los centros turísticos de la región, ya que un porcentaje de personas que visitan la ciudad indican no tener información alguna del turismo en Tacna.

\section{REFERENCIAS}

1. Constitución Política del Perú, 1993.

2. Hernández Sampieri, R. / Fernández Collado, C. / Baptista Lucio, Pilar. 1997, Metodología de la investigación, McGraw-Hill Interamericana de México.

3. Ley General del Turismo Nro.29408. 18 de Setiembre del 2009.

4. Plan Maestro de Desarrollo Turístico Nacional en la República del Perú. 1999.

5. MONITOR COMPANY. 1994. Construyendo ventajas competitivas en el Perú. Lima: Ministerio de Industria, Turismo, Integración y Negociaciones Comerciales Internacionales.

6. YAMADA, G.; CHACALTANA, J. 2007. Documento de discusión: Generación de empleo en el Perú: Seis casos recientes de éxito. Lima: Centro de Investigación de la Universidad del Pacífico.

\section{Páginas Web:}

- Ministerio de Comercio Exterior y Turismo: www.mincetur.gob.pe.

- Comisión para la Promoción de la Exportación y el Turismo PROMPERÚ.

- www.promperu.gob.pe

- http://www.world-tourism.org; información detallada sobre las actividades y los informes de la Organización Mundial del Turismo (OMT). 